Canad. Math. Bull. Vol. 21 (3), 1978

\title{
A CHARACTERIZATION OF SOFT HYPERGRAPHS
}

\author{
BY
}

PETER J. SLATER*

\begin{abstract}
A hypergraph $H=(X, \mathscr{E})$ is a subtree of a tree (SOFT) hypergraph if there exists a tree $T$ such that $X=V(T)$ and for each $E_{i} \in \mathscr{E}$ there is a subtree $T_{i}$ of $T$ such that $E_{i}=V\left(T_{i}\right)$. It is shown that $H$ is a SOFT hypergraph if and only if $\mathscr{E}$ has the Helly property and $\Omega(\mathscr{E})$, the intersection graph of $\mathscr{E}$, is chordal. Results of Berge and Gavril have previously shown these to be necessary conditions.
\end{abstract}

The couple $H=(X, \mathscr{E})$ is a hypergraph if $X$ is a finite set and $\mathscr{E}=\left\{E_{i}: i \in I\right\}=$ $\left\{E_{1}, E_{2}, \ldots, E_{m}\right\}$ is a family of subsets of $X$ (called edges) with each $E_{i} \neq \phi$ and $\bigcup_{i \in I} E_{i}=X$. A family $\mathscr{E}_{0} \subseteq \mathscr{E}$ is a matching if the edges of $\mathscr{E}_{0}$ are pairwise disjoint, and $v(H)$ denotes the maximum cardinality of a matching of $H$; a subset $X_{0} \subseteq X$ is a transversal if $X_{0} \cap E_{i} \neq \phi$ for each $i \in I$, and $\tau(H)$ denotes the minimum cardinality of a transversal of $H$. Clearly $v(H) \leq \tau(H)$, and if $v(H)=\tau(H)$ then $H$ is said to be a Menger system.

THEOREM 1. [2] Let $V(T)$ be the vertex set of a tree $T$ and $H$ be a hypergraph with $\mathscr{E}=\left\{S_{1}, \ldots, S_{m}\right\}$, where each $S_{i}$ is the vertex set of a subtree of $T$, and with $X=\bigcup_{i=1}^{t} S_{i}$. Then $H$ is a Menger system.

Call hypergraph $H=(X, \mathscr{E})$ a subtree of a tree (SOFT) hypergraph if there exists a tree $T$ such that $X=V(T)$ and for each $E_{i} \in \mathscr{E}$ there is a subtree $T_{i}$ of $T$ such that $E_{i}=V\left(T_{i}\right)$. The objective here is a characterization of SOFT hypergraphs. If the connected components of $H$ are $H_{1}=\left(X_{1}, \mathscr{C}_{1}\right), \ldots, H_{c}=$ $\left(X_{c}, \mathscr{E}_{c}\right)$ then clearly $v(H)=v\left(H_{1}\right)+\cdots+v\left(H_{c}\right), \tau(H)=\tau\left(H_{1}\right)+\cdots+\tau\left(H_{c}\right), H$ is a Menger system if and only if each $H_{i}$ is, and $H$ is a SOFT hypergraph if and only if each $H_{i}$ is.

It is said that $\mathscr{E}$ satisfies the Helly property if $J \subseteq I$ and $E_{i} \cap E_{i} \neq \phi$ for all $i, j \in J$ implies that $\bigcap_{j \in J} E_{j} \neq \phi$.

THEOREM 2. [1, p. 399, Example 3]. If $H$ is a SOFT hypergraph then $\mathscr{E}$ satisfies the Helly property.

Received by the editors January 25, 1977.

Key Words: chordal graph; Helly property; hypergraph; Menger system; tree.

AMS-MOS 1970 Subject Classification: 05005

* This work was supported by the U.S. Energy Research and Development Administration (ERDA), Contract No. AT(29-1)-789. By acceptance of this article, the publisher and/or recipient acknowledges the U.S. Government's right to retain a non-exclusive, royalty-free license in and to any copyright covering this paper. 
Let $\Omega(\mathscr{E})$ denote the intersection graph on $\mathscr{E}$, that is, the vertex set of $\Omega(\mathscr{E})$ is $\left\{e_{1}, e_{2}, \ldots, e_{m}\right\}$ with $\left(e_{i}, e_{j}\right)$ an edge of $\Omega(\mathscr{E})$ if and only if $E_{i} \cap E_{j} \neq \phi$. A graph is called a chordal graph if every circuit with more than three vertices has a chord (an edge connecting two non-consecutive vertices of the circuit), and a graph is called a subtree graph if it is the intersection graph of a family of subtrees of a tree. Gavril [4] has shown that a graph is a subtree graph if and only if it is a chordal graph. For example, if $H=(X, \mathscr{E})$ with $X=$ $\{1,2,3,4,5,6\}$ and $\mathscr{E}=\left\{E_{1}, \ldots, E_{6}\right\}$ where $E_{1}=\{1,2\}, E_{2}=\{1,3\}, E_{3}=\{1,5\}$, $E_{4}=\{3,4\}, E_{5}=\{3,5\}$ and $E_{6}=\{5,6\}$, then $\Omega(\mathscr{E})$ is chordal and hence a subtree graph. Note that being a chordal graph is the property of an unlabelled graph. Indeed, no tree can have subtrees whose vertex sets are $E_{1}, \ldots, E_{6}$, and $H$ is not a SOFT hypergraph, as can be seen by observing that $\{\{1,3\},\{1,5\},\{3,5\}\}$ shows that $\mathscr{E}$ does not have the Helly property. One does, however, obtain the following theorem as a corollary of Gavril's result.

TheOREM 3. If $H$ is a SOFT hypergraph, then $\Omega(\mathscr{E})$ is chordal.

THEOREM 4. Hypergraph $H=(X, \mathscr{E})$ is a SOFT hypergraph if and only if $\Omega(\mathscr{E})$ is chordal and $\mathscr{E}$ satisfies the Helly property.

Proof. By Theorems 2 and 3 the conditions are necessary if $H$ is a SOFT hypergraph.

For the converse, induct on $m=|\mathscr{E}|$. Clearly if $m=1$, one can select any tree $T$ with $\left|E_{1}\right|$ vertices labelled with the elements of $E_{1}$. For $m=2$ one can form trees $T_{1}, T_{2}$ and $T_{3}$ with vertex sets $E_{1} \cap E_{2}, E_{1}-E_{2}$ and $E_{2}-E_{1}$ (any one, or the last two, of which may be empty). Select vertices $v_{1}, v_{2}$ and $v_{3}$ in $T_{1}, T_{2}$ and $T_{3}$, respectively, and form tree $T$ by adding edges $\left(v_{1}, v_{2}\right)$ and $\left(v_{1}, v_{3}\right)$. If $E_{1} \cap E_{2}=\phi$, then one adds edge $\left(v_{2}, v_{3}\right)$.

Suppose $m \geq 3$ and, by induction, that the conditions are sufficient if $|\mathscr{E}| \leq$ $m-1$. Since $\Omega(\mathscr{E})$ is chordal it has a vertex for which any two vertices adjacent to it are adjacent to each other (see [3] or [5]). Thus it can be assumed that $e_{1}$ is such a vertex, $e_{i}$ is adjacent to $e_{1}$ (where $2 \leq i \leq m$ ) if and only if $2 \leq i \leq k$ (if $e_{1}$ is an isolated vertex then one is clearly done by induction, and so one assumes $2 \leq k$ ), and any two of $e_{1}, e_{2}, \ldots, e_{k}$ are adjacent. Since $\mathscr{E}$ satisfies the Helly property, one has $\bigcap_{i=1}^{k} E_{i} \neq \phi$, say $a \in \bigcap_{i=1}^{k} E_{i}=E$. Let $E_{1}^{\prime}=E_{1}-a$. For $2 \leq i \leq m$, let $E_{i}^{\prime}=E_{i}-E_{1}^{\prime}$.

Now suppose $2 \leq h<j \leq m$. Since $E_{i}^{\prime} \subseteq E_{i}$, if $E_{h}^{\prime} \cap E_{j}^{\prime} \neq \phi$ then $E_{h} \cap E_{j} \neq \phi$. Assume $E_{h} \cap E_{j} \neq \phi$. If $j \geq k+1$ then, since $E_{j} \cap E_{1}=\phi$, one has $E_{h}^{\prime} \cap E_{j}^{\prime}=$ $E_{h}^{\prime} \cap E_{j}=E_{h} \cap E_{j} \neq \phi$; if $j \leq k$ then $a \in E_{h}^{\prime} \cap E_{j}^{\prime}$. Thus $E_{h} \cap E_{j} \neq \phi$ if and only if $E_{h}^{\prime} \cap E_{j}^{\prime} \neq \phi$. This implies that $\mathscr{E}^{\prime}=\left\{E_{2}^{\prime}, \ldots, E_{m}^{\prime}\right\}$ has the Helly property and that $\Omega\left(\mathscr{E}^{\prime}\right)$, which is isomorphic to $\Omega(\mathscr{E})-e_{1}$, is chordal. By induction, $H^{\prime}=$ $\left(X-E_{1}^{\prime}, \mathscr{E}^{\prime}\right)$ is a SOFT hypergraph of some tree $T^{\prime}$.

Let $T$ be obtained from $T^{\prime}$ by adding $\left|E_{1}^{\prime}\right|$ vertices, labelled with the elements 
of $E_{1}^{\prime}$, each of which is made adjacent to $a$. It is straightforward to see that $E_{i}$ is the vertex set of a subtree of tree $T$ for $1 \leq i \leq m$ and that the vertex set of $T$ is $X$.

\section{REFERENCES}

1. C. Berge, Graphs and Hypergraphs, North-Holland, Amsterdam, 1973.

2. E. J. Cockayne, S. T. Hedetniemi and P. J. Slater, Matchings and Transversals in Hypergraphs, Domination and Independence in Trees, to appear in JCT(B).

3. G. A. Dirac, On rigid circuit graphs, Abh. Math. Sem. Univ. Hamburg 25, 71-76 (1961).

4. F. Gavril, The Intersection Graphs of Subtrees in Trees Are Exactly the Chordal Graphs, JCT(B) 16, 47-56(1974).

5. D. J. Rose, Triangulated Graphs and the Elimination Process, J. Math. Anal. Appl. 32, 597-609(1970).

Applied Mathematics Division 5121

SANDIA LABORATORIES

Albuquerque, NeW Mexico 87115 